\title{
De Paris en Provence, circulation et réception du livre italien à la première modernité
}

Da Parigi alla Provenza, circolazione e ricezione del libro italiano nella prima età moderna

From Paris to Provence, Circulation and Reception of the Early Modern Italian Book

\section{Amélie Ferrigno}

\section{OpenEdition Journals}

Édition électronique

URL : http://journals.openedition.org/cei/7848

DOI : $10.4000 /$ cei.7848

ISSN : 2260-779X

Éditeur

UGA Éditions/Université Grenoble Alpes

Édition imprimée

ISBN : 978-2-37747-225-3

ISSN : $1770-9571$

Référence électronique

Amélie Ferrigno, « De Paris en Provence, circulation et réception du livre italien à la première modernité », Cahiers d'études italiennes [En ligne], 31 | 2020, mis en ligne le 06 octobre 2020, consulté le 28 mars 2021. URL : http://journals.openedition.org/cei/7848 ; DOl : https://doi.org/10.4000/cei. 7848

Ce document a été généré automatiquement le 28 mars 2021. 


\section{De Paris en Provence, circulation et réception du livre italien à la première modernité}

Da Parigi alla Provenza, circolazione e ricezione del libro italiano nella prima età moderna

From Paris to Provence, Circulation and Reception of the Early Modern Italian Book

Amélie Ferrigno

\section{Introduction}

De par sa configuration géographique et les fluctuations de ses frontières avec le pays transalpin, la Provence fut historiquement un territoire d'échanges (commerciaux, intellectuels, linguistiques, artistiques...) où l'Italie a laissé de nombreuses empreintes. Ainsi, le premier livre en langue d'oc - plus précisément, en provençal niçois Lo Compendion de l'Abaco de Francès Pellos est imprimé à Turin en 1492, faisant de ce traité de mathématique pour commerçant un témoin privilégié du dynamisme commercial et du métissage culturel dans cet espace ${ }^{1}$. Si la présence et la production de savants et intellectuels italiens en Provence, tout autant que les réseaux d'échanges et de correspondances avec leurs homologues provençaux au Moyen Âge et à la Renaissance ont été largement étudiés ${ }^{2}$, le circuit de l'édition en langue italienne en Provence reste quant à lui à préciser. Malgré l'évidente circulation des hommes, on déplore, en effet, le peu d'études consacrées à la diffusion du livre italien à la Renaissance dans ce territoire ${ }^{3}$. Avait-il un circuit spécifique, de par la proximité géographique? Ou bien a-t-il été exclusivement diffusé par les canaux propres aux cercles savants, notamment parisiens ou lyonnais ? La Provence a-t-elle été une région de transit de livres italiens vers d'autres régions ou pays? Quel était le profil des possesseurs de livres en langue italienne en Provence? 
2 Dans le cadre du projet ANR EDITEF ${ }^{4}$ qui étudie la diffusion du livre italien dans l'espace francophone durant la première modernité, de grandes enquêtes ont été menées au sein des fonds italiens de plusieurs bibliothèques de France et ont permis de préciser - grâce aux relevés des ex-libris et autres marques de provenance au sein des ouvrages

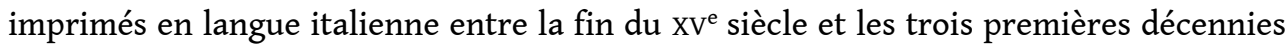
$\mathrm{du} \mathrm{XVII}^{\mathrm{e}}$ siècle - les principaux circuits de diffusion du livre italien et de mesurer l'impact de leur pénétration dans les grands centres de réceptions francophones 5 .

3 Cette enquête reste à poursuivre au sein des collections provençales, car l'examen systématique des provenances n'y a encore jamais été réalisé, afin d'observer les particularités de la circulation et de la réception du livre italien en Provence à partir du $\mathrm{XVI}^{\mathrm{e}}$ siècle. On présentera ici les premiers résultats de cette enquête, toujours en cours, à partir des données fournies par deux fonds aixois: les fonds italiens de la bibliothèque Méjanes et de la bibliothèque du Musée Paul Arbaud. Le relevé des exlibris et autres marques de provenance au sein des ouvrages imprimés en langue italienne entre le $\mathrm{xv}^{\mathrm{e}}$ et le $\mathrm{xVII}^{\mathrm{e}}$ siècle constituent les premières données, qui s'inscrivent dans un questionnement plus large sur la réception du livre italien dans cette région. Ces deux fonds aixois, rassemblés par deux collectionneurs appartenant à deux époques différentes, révèlent les premières pistes d'acheminement du livre italien jusqu'en Provence à travers la chronologie des divers possesseurs relevés. Ils montrent également des pratiques similaires d'acquisition chez Méjanes et Arbaud, qui font de Paris une étape d'approvisionnement incontournable dans la constitution des bibliothèques de Province. Dans la perspective d'une compréhension globale du parcours du livre en langue italienne dans l'espace francophone à la Renaissance, ces données seront observées au regard d'autres études, notamment des résultats de l'enquête menée à la bibliothèque Mazarine, qui grâce à son large corpus a permis de dévoiler les principaux canaux de diffusion du livre italien à Paris.

\section{Le fonds italien de la bibliothèque Méjanes}

4 Le fonds initial de la bibliothèque de Jean-Baptiste Marie de Piquet (1729-1786), marquis de Méjanes, collectionneur, érudit féru d'histoire, a été constitué durant la seconde moitié du XVIII ${ }^{e}$ siècle, entre 1765 et 1786, et se composait d'environ 80000 ouvrages $^{6}$. Le corpus des livres italiens compte environ sept cents ouvrages en langue italienne imprimés entre la fin du $\mathrm{Xv}^{\mathrm{e}}$ siècle et les trois premières décennies du XVII siècle. L'analyse des provenances relevées est toujours en cours, cependant les premières marques de provenance identifiées permettent de faire quelques observations sur les circuits empruntés par ces livres.

5 En plus des libraires aixois, notamment les David, à qui il passe commande, on sait que le marquis de Méjanes fréquentait les ventes publiques de Paris et qu'il entretenait une correspondance avec divers libraires parisiens et étrangers auprès desquels il acquérait ses livres, comme en attestent ses propres notes manuscrites portant sur les particularités d'édition et signalant les mentions d'achat, que l'on retrouve sur les pages de garde de ses ouvrages («acheté à Paris [...] à la vente de $\mathrm{Mr}$. Ra[n]don de Goisset $n^{\circ} 1341$ ", " acheté [...] à Paris à une vente faite à Hotel de Gullion le 13 [...] 1784 », ou « Paris, quai des augustins, $1783^{7}$ », ou encore, la mention d'une acquisition faite lors d'une vente de livres italiens organisée par René François Fétil $\left.{ }^{8}\right)$. D’après l'échantillon des livres examinés, nous remarquons qu'en réalité peu de ces ouvrages 
en langue italienne proviennent de librairies aixoises. La mention des libraires David n'a été relevée que trois fois dans un ensemble correspondant à la moitié du corpus, ce qui est plutôt étonnant car on sait que ces derniers avaient des correspondants libraires en Italie, notamment à Gênes, à Venise et à Rome ${ }^{9}$. La plupart des éditions italiennes du corpus de la Méjanes semblent donc provenir de ventes parisiennes, ou d'acquisitions auprès de libraires parisiens, ou lyonnais ${ }^{10}$. On observe, dans de nombreux exemplaires, la présence de plusieurs ex-libris désignant un premier possesseur italien, vraisemblablement $\mathrm{du} \mathrm{XVI}^{\mathrm{e}}$ siècle, correspondant à une première période de circulation au sein de la péninsule italienne proche de la date d'édition de l'ouvrage, puis un possesseur français plus récent, ce qui permet de déduire (ou si le lieu d'achat est mentionné dans l'ex-libris, d'avoir la preuve) que le livre a ensuite traversé la frontière jusqu'en France, et enfin, la mention d'achat du marquis de Méjanes $^{11}$.

6 Ces informations témoignent d'un premier circuit des livres italiens de la collection du marquis que l'on peut rapprocher d'un des circuits retracés par l'enquête menée au sein du fonds italien de la bibliothèque Mazarine. En dehors des caractéristiques spécifiques à l'histoire de la constitution du fonds de la bibliothèque de Mazarin, notamment par son bibliothécaire Gabriel Naudé $^{12}$, ce corpus offrant un large panorama de l'édition italienne (plus de 4000 ouvrages imprimés en langue italienne, des incunables jusqu'à 1630) a permis de tracer les grandes lignes de la circulation du livre italien à Paris à la première modernité. L'examen de ces ouvrages ${ }^{13}$ a montré que les livres italiens provenant des achats de Naudé (environ 700 exemplaires du corpus reconnaissables grâce à plusieurs croisements de données, numéros d'inventaires, reliure caractéristique) contiennent peu de marques de provenance, et que la plupart des ex-libris sont majoritairement italiens (dont une grande partie d'ex-libris italiens non identifiés). Ces données confirment ainsi la thèse d'un circuit du livre assez court pour ces ouvrages, que Naudé vient chercher directement en Italie peu de temps après leur impression. Les ouvrages du corpus de la Mazarine issus de récupérations révolutionnaires contiennent quant à eux plus de marques de provenance. On y relève souvent, comme cela a pu être observé dans les ouvrages italiens du fonds de la bibliothèque Méjanes, les ex-libris de possesseurs italiens non identifiés appartenant vraisemblablement au $\mathrm{XVI}^{\mathrm{e}}$ siècle, puis une marque de provenance française plus récente (généralement du XvII ${ }^{\mathrm{e}}$ siècle) d'un possesseur privé ou d'une institution. Cette similitude dans la chronologie des possesseurs, par rapport aux provenances relevées au sein du fonds italien du marquis de Méjanes, s'explique logiquement par le fait que le marquis ait acquis une grande partie de ces ouvrages auprès de libraires parisiens, possédant donc des livres italiens ayant vraisemblablement suivi le même circuit que certains livres retrouvés au sein de la bibliothèque Mazarine.

7 Il est difficile de déceler dans ces ouvrages des caractéristiques qui seraient propres à la circulation du livre italien en Provence avant leur acquisition par Jean-Baptiste Marie de Piquet. Néanmoins, d'autres marques de provenance relevées au sein du fonds italien de la bibliothèque Méjanes indiquent un second circuit qui se développait quant à lui dans l'est de la France, du côté de Lyon et des régions italiennes limitrophes. Nous relevons, par exemple, sur un même exemplaire trois mentions d'achats différentes en l'espace d'une vingtaine d'années et une circulation assez dense du livre italien entre Gênes et Lyon («1558 di luglio comprato in Genoa », « a 6 di luglio del 1564 In Lione », « 1573 di 
febraio comprato in Monlunello $\left.{ }^{14} »\right)$, tandis qu'un autre exemplaire contient l'ex-libris d'un possesseur lyonnais, un certain « Claudio maestro lionese ${ }^{15}$ ».

Il est difficile d'avoir une estimation précise du nombre de livres italiens qui figuraient dans la bibliothèque originelle du marquis. Cependant, la plupart des 700 ouvrages en langue italienne du fonds de la bibliothèque Méjanes montrent des caractéristiques communes permettant de les rattacher au fonds initial, ensuite légué aux États de Provence en 1786, notamment les signets que l'on retrouve dans presque tous les exemplaires et les fameuses notes manuscrites de Méjanes relatives à la particularité de l'édition, que l'on peut lire sur la garde supérieure des ouvrages, avec le plus souvent, le nom du libraire, le lieu et la date d'acquisition, ainsi que le prix de l'ouvrage. Ces mentions sont des sources importantes pour la connaissance du réseau libraire du marquis de Méjanes et livrent des informations complémentaires sur le circuit du livre italien, mais également sur les particularités d'édition recherchées et le genre de livres constituant sa collection.

9 La collection du marquis de Méjanes ne démontre pas une recherche spécifique de livres italiens, mais correspond à celle d'un collectionneur de livres, à la manière des grands aristocrates européens de son temps, ou du moins, des grands aristocrates français ou des grands financiers parisiens de la seconde moitié du xvIII ${ }^{\mathrm{e}}$ siècle. Elle s'inscrit dans une tradition bibliophilique qui remonte au xvII ${ }^{\mathrm{e}}$ siècle, avec ses différents modèles de bibliothèques, principalement la bibliothèque de l'honnête homme, particulièrement fournie en histoire, histoire des lettres, et dont l'une des règles est de constituer une partie consacrée aux éditions $d u x^{e}$ et $d u x^{2} I^{e}$ siècle, en faisant l'acquisition de livres anciens, ou plus justement, qui témoignent d'un état du savoir ancien, par rapport à son époque ${ }^{16}$. Le marquis de Méjanes est donc à la recherche d'éditions rares, particulières, et applique également ce critère aux éditions en langue italienne.

10 Les ouvrages italiens de ce corpus sont pour la plupart des livres d'histoire, conformément à la démarche bibliophilique adoptée au temps du marquis (Historia di Fiandra, Historia della guerra sacra di Gierusalemme, Historia di Bologna, Historia della guerra fra turchi e persiani...), puis des livres de littérature (L'Orlando furioso de l'Arioste, des œuvres du Tasse, de Dante, de Pétrarque ou de Boccace). On retrouve ainsi les ouvrages italiens qui composaient les bibliothèques des possesseurs français du XVI ${ }^{\mathrm{e}}$ et du XVII siècle, axés sur l'apprentissage de la langue, et reflétant les débats de l'époque sur la forme idéale de la langue italienne (Carlo Lenzoni, Difesa della lingua fiorentina e di Dante; Pierfrancesco Giambullari, Origine della lingua fiorentina...). Le corpus en langue italienne est également composé de quelques livres de science, et d'ouvrages spécifiques de l'édition italienne de la Renaissance, comme les livres de divertissement ou de culture contemporaine (Giovanni Bardi, Discorso sopra il calcio fiorentino; Tommaso Garzoni, Piazza universale di tutte le professioni del mondo...). Conformément aux pratiques des collectionneurs et bibliophiles de son époque, le marquis de Méjanes recherchait des documents rares ou précieux, des particularités d'éditions bibliographiques, historiques ou littéraires - et probablement certaines reliures - comme en témoignent les longues notes laissées sur les gardes, dans lesquelles il indique qu'il s'agit d'une "bonne et rare édition ", d'une "bonne édition recherchée », ou d'une " édition rare ».

11 Les livres italiens du marquis de Méjanes représentent donc un pan de sa bibliothèque, qui ne reflète pas un intérêt spécial pour l'Italie ou l'édition italienne, mais la nécessité 
de trouver des éditions témoignant d'une étape historique dans le savoir. Les éditions et les auteurs italiens du $\mathrm{XVI}^{\mathrm{e}}$ siècle apparaissent ainsi comme une composante essentielle à la constitution de sa bibliothèque en tant que marque caractéristique du savoir à la Renaissance.

\section{Le fonds italien de la collection Paul Arbaud}

12 Le circuit d'acquisition passant par un réseau de relieurs et libraires parisiens ou lyonnais est également repérable dans le fonds de la bibliothèque de Paul Arbaud (1831-1911), collectionneur aixois qui abritait dans son hôtel particulier, livres, manuscrits et objets d'art formant une des plus riches collections consacrées à la Provence, qu'il mettait à disposition de ses amis. La célèbre Arbaudenco, comme on appelait alors le musée bibliographique et archéologique Paul Arbaud, connue des érudits provençaux mais également des bibliophiles parisiens $\mathrm{du} \mathrm{XIX}^{\mathrm{e}}$ et $\mathrm{du} \mathrm{Xx}^{\mathrm{e}}$ siècle, était vraisemblablement fréquentée par la communauté savante, des membres du Félibrige, des académiciens (citons, parmi les contemporains de Paul Arbaud, Gaston de Saporta, Frédéric Mistral, Léon de Berluc-Pérussis, Charles de Ribbe...). Alors que les productions scientifiques sur le fonds manquent cruellement, puisqu'il n'existe quasiment aucune étude sur l'histoire de la collection Arbaud (musée et bibliothèque) ${ }^{17}$, ni de biographie consacrée à Paul Arbaud, pas plus que de réelles études sur la constitution de sa bibliothèque, l'entreprise de catalogage informatisé des imprimés de la bibliothèque qui a débuté en $2015^{18}$ permet de redécouvrir l'héritage que Paul Arbaud a laissé à l'Académie des Sciences, Agriculture, Arts et Belles-Lettres d'Aix - dont il était membre d'honneur - devenue propriétaire des lieux et de la collection, et siégeant au sein de l'hôtel Paul Arbaud, dans le quartier Mazarin d'Aix-en Provence depuis $1912^{19}$.

13 Les inventaires et fichiers (non exhaustifs) auxquels s'ajoute l'avancée du catalogage informatisé laissent entrevoir une collection fournie dans tous les genres. La bibliothèque de Paul Arbaud est avant tout celle d'un amoureux de la Provence, à laquelle il rend hommage, mais elle reflète également les pratiques d'un collectionneur type du XIX siècle, avec une recherche scrupuleuse d'éditions rares (nombreux tirages limités, particularités d'édition...) et une attention spéciale accordée aux reliures d'exception qu'il acquiert ou qu'il fait faire en s'adressant directement aux grands relieurs parisiens (Ruban, Thompson, Duru, Bauzonnet, Cottin-Simier, Gruel, Koehler, Meunier...). Au-delà du livre religieux qui représente une grande partie de la collection, fait courant dans la bibliothèque d'un homme du xixe siècle, la richesse de la bibliothèque Arbaud tenait vraisemblablement à sa diversité. La littérature contemporaine y est, en effet, particulièrement bien représentée, avec, entre autres : une reproduction en fac-similé du manuscrit de François Coppée, Le passant, contenant un portrait de Sarah Bernhardt (1897) ; un tirage limité de Henry Monnier, Les bas-fonds de la société, avec dessins à la plume de Félicien Rops; les œuvres de Charles Poncy, poète provençal et «maçon de Toulon» soutenu par Georges Sand; un exemplaire portant la mention « imprimé pour M. P. Arbaud » d'une édition limitée de À rebours de J.-K. Huysmans $(1903)^{20}$. Ces livres sont le reflet de la vie littéraire et intellectuelle de son époque et dessinent le profil d'un collectionneur à la page des courants avantgardistes de son temps. Ils côtoient désormais sur les étagères des volumes sur l'histoire du livre en Provence, qui constituent sans aucun doute le cœur de la 
bibliothèque Arbaud. Toutes les grandes étapes de l'histoire de la littérature provençale y sont représentées. Malgré l'absence de l'ouvrage de Francès Pellos cité plus haut, connu comme le premier livre imprimé en provençal ${ }^{21}$, on y trouve la plupart des éditions marquantes de la littérature provençale : les éditions en italien macaronique d'Antoine Arena, les premières éditions des œuvres de Louis Bellaud de la Bellaudière, de Claude Brueys..., le premier livre imprimé à Aix ${ }^{22}$, jusqu'aux ouvrages de Frédéric Mistral, dont certains présentent des ex-dono de l'auteur témoignant d'une forte amitié entre les deux hommes. La bibliothèque contient également de nombreux ouvrages de géographie (ou guides de la Provence...) et d'histoire des principales villes de Provence, la spécificité de la bibliothèque étant de présenter une collection axée sur l'histoire de la Provence et les œuvres d'auteurs provençaux; particularité que l'on retrouve également au sein du corpus de livres en langue italienne.

Parmi ces éditions rares, environ une quarantaine de livres italiens imprimés entre le $\mathrm{xVI}^{\mathrm{e}}$ et le $\mathrm{XVII}^{\mathrm{e}}$ siècle ont été relevés à ce jour, comprenant des traductions italiennes des œuvres de Michel Nostradamus et de son frère Jean ${ }^{23}$, ou encore des Métamorphoses d'Ovide ${ }^{24}$. Figurent également quelques classiques de la littérature italienne avec un exemplaire de Dante ${ }^{25}$, une édition de l'Orlando furioso ${ }^{26}$, les Lettere d'Isabella Andreini ${ }^{27}$, ou encore une édition de Battista Guarini ${ }^{28}$. La bibliothèque contient également un fonds dédié à Pétrarque, avec des éditions latines et des traductions françaises sans doute à rattacher à une partie consacrée à la Fontaine de Vaucluse ${ }^{29}$. Parmi les ouvrages d'histoire des villes de Provence, on trouve une histoire de la cité d'Avignon traduite en italien ${ }^{30}$. Enfin, 16 ouvrages en langue italienne sur le culte de sainte Marie-Madeleine ${ }^{31}$, à qui l'on doit l'évangélisation de la Provence et à laquelle Arbaud consacre une belle partie de sa collection, dont 6 exemplaires sont des pièces de théâtre et de la poésie magdalénienne, spécialité d'un groupe de poètes aixois ${ }^{32}$.

remarquons que certains ouvrages en langue italienne ne présentent aucun lien thématique avec la Provence, ni une quelconque particularité d'édition, en dépit des critères d'acquisitions de Paul Arbaud. Leur présence au sein de la bibliothèque consacrée " à la gloire de la Provence ${ }^{33}$ " semble alors se justifier non par les spécificités d'édition, ni le sujet de l'ouvrage, mais par les ex-libris qu'ils contiennent. Notons, par exemple, la présence d'un exemplaire des Lettere di tredici huomini raccolte da Tommaso Porcacchi (Venise, 1554) contenant l'ex-libris « di Bartolomeo Arnigio", auteur italien du XvI ${ }^{\mathrm{e}}$ siècle dans la veine de Pétrarque et admirateur du poète ${ }^{34}$. L'ouvrage Dichiarazioni della pianta dell'antiche Siracuse e d'alcune scelte medaglie d'esse de Vincenzo Mirabella (Naples, 1613) porte quant à lui, outre l'ex-libris de l'architecte lyonnais Antoine Marie Chenavard, le chiffre de Peiresc, célèbre érudit aixois d'origine italienne ${ }^{35}$. Autre exemple, l'ouvrage de Lodovico Melzo, Regole militari sopra il governo e servitio particolare della cavalleria (Anvers, 1611) porte sur les plats les armes de la famille Forbin (d'or au chevron d'azur accompagnée de trois têtes de léopards de sable, arrachées, de gueules), une des plus célèbres familles de la noblesse provençale ${ }^{36}$.

Cet échantillon italien de la bibliothèque Paul Arbaud révèle certains critères et pratiques bibliophiliques du collectionneur (thèmes, particularités et rareté des éditions, recherche d'ex-libris provençaux...), en plus de présenter, une fois de plus, les exemplaires de l'édition italienne $\mathrm{du} \mathrm{xvI}^{\mathrm{e}}$ siècle qui ont le plus circulé. Incontestablement très proche du milieu bibliophile parisien, comme en atteste ses livres de comptes, ou l'examen des reliures, Paul Arbaud s'approvisionnait, à l'instar du marquis de Méjanes, auprès de libraires ou marchands de la capitale. Bien que le corpus 
Arbaud soit trop petit pour apporter des données significatives sur la circulation du livre italien en Provence, cet échantillon comporte cependant au moins - car tout le fonds n'a pas encore été exploré - deux marques de provenance démontrant une circulation du livre italien parmi la communauté scientifique et littéraire en Provence durant la première modernité, avec les ex-libris d'Arnigio et de Peiresc, et confirme que le livre italien était bien présent dans les bibliothèques de la noblesse provençale avec l'ex-libris de la famille Forbin.

\section{Conclusion}

La diffusion du livre italien en dehors de la péninsule commence relativement tôt après les débuts de l'imprimerie en Italie vers 1465. Les grandes études consacrées au livre italien dans les bibliothèques françaises $\mathrm{du} \mathrm{xvI}^{\mathrm{e}}$ siècle soulignent la difficulté à le quantifier au sein des collections, et n'ont pu constater qu'une présence assez faible à partir des rares inventaires publiés. Nous pouvons néanmoins estimer à $10 \%$, en moyenne, la présence de livres en langue italienne au sein de grandes bibliothèques de bibliophiles français de la fin du $\mathrm{XVI}^{\mathrm{e}}$ et du début XVII ${ }^{\mathrm{e}}$ siècle, en nous appuyant sur les relevés effectués au sein de quelques inventaires ${ }^{37}$. Le livre italien et l'intérêt pour la langue et la culture italiennes apparaissent comme une constante dans les bibliothèques françaises dès le $\mathrm{xvI}^{\mathrm{e}}$ siècle, dans tous les domaines, ainsi qu'auprès de tous types de possesseurs (nobles, érudits, officiers, financiers...) durant cette époque. L'enquête menée au sein de la bibliothèque Mazarine avait confirmé que le développement d'un "italianisme bibliophilique ", qui débute au XVI e siècle, s'accentue durant le XVII ${ }^{e}$ siècle avec un nombre croissant de possesseurs français, qui adoptent tous, à en juger par les thématiques des livres, des pratiques quasi similaires, au-delà de quelques nuances à relever, notamment pour les hommes d'Église ${ }^{38}$. Leur recherche d'ouvrages en italien semble dominée par le besoin d'un apprentissage de la langue, avec de nombreux livres de vocabulaire, de grammaire, des éditions bilingues et les incontournables classiques de la littérature italienne qui constituent la plus grande partie des corpus (à côté des livres d'histoire, de géographie, de quelques traités scientifiques, et de genres plus spécialisés comme les livres de comptabilité marchande, de divertissement, ou de culture contemporaine...) et qui font office de véritables manuels au travers desquels la culture et la langue italienne se diffusent auprès des lecteurs.

La diffusion du livre italien dans les grands centres de réception tels que Paris ou Lyon correspond à des besoins et à un contexte précis. À Paris, elle semble principalement liée aux activités de la cour, et se mêle à un italianisme artistique, littéraire et éditorial qui se développe depuis le $\mathrm{XvI}^{\mathrm{e}}$ siècle. De par son histoire et sa proximité géographique avec l'Italie, on peut supposer que le livre italien se soit diffusé différemment en territoire provençal, mais cette spécificité reste encore à définir, en poursuivant l'enquête au sein des grandes collections privées et publiques de Provence pour connaître plus précisément les canaux de diffusion du livre italien jusqu'en Provence à la Renaissance, le profil des possesseurs, le genre de livre recherché, et d'avoir une meilleure idée de la façon dont la Provence percevait ses voisins italiens. En effet, les premières données recueillies au sein des deux fonds aixois sont plutôt cohérentes avec les résultats d'enquêtes précédentes menées au sein d'autres fonds francophones, aussi bien dans le repérage d'une chronologie des possesseurs selon leur nationalité qui 
révèle les traces de la circulation des livres, que par l'histoire de la constitution des fonds par les collectionneurs plus récents. Le début de l'enquête sur le territoire provençal fait ainsi émerger de nouvelles questions quant à l'acheminement du livre italien en Provence $a u x I^{e}$ et au xvII siècle, et au rôle de Paris ou Lyon dans la constitution des collections de Province.

Les possesseurs français des $\mathrm{XVI}^{\mathrm{e}}$ et $\mathrm{xVII}{ }^{\mathrm{e}}$ siècles ont majoritairement associé leur recherche d'éditions italiennes à un usage pratique répondant aux besoins diplomatiques ou culturels du moment, et à une tradition littéraire, un héritage des Lettres italiennes et un goût pour l'Italie qui s'est développé en France depuis François $I^{e r}$. Les siècles passant, l'édition italienne du $\mathrm{XVI}^{\mathrm{e}}$ est davantage recherchée pour ses particularités et répond à d'autres préoccupations bibliophiliques. Une image de l'Italie émerge de façon récurrente des fonds étudiés : l'Italie recherchée à travers les livres est l'Italie savante, l'Italie de l'humanisme et de la quête des savoirs, celle des grands écrivains, artistes et penseurs qui ont fait la gloire de l'Italie de la Renaissance.

\section{BIBLIOGRAPHIE}

ADAM Renaud et LASTRAIOLI Chiara (éd.), Itinéraires du livre italien à la Renaissance : Suisse romande, anciens Pays-Bas et Liège, Paris, Garnier, coll. « Travaux du Centre d'études supérieures de la Renaissance », 2019.

BALDACCHINI Lorenzo, Il libro e le sue reti, Bologne, Bononia University Press, coll. « Studi sul patrimonio culturale », 2015.

BALSAMo Jean, L'amorevolezza verso le cose italiche. Le livre italien à Paris au XvI ${ }^{e}$ siècle, Genève, Librairie Droz, coll. « Travaux d'humanisme et Renaissance », 2015.

BALSAMO Jean, Les rencontres des muses : italianisme et anti-italianisme dans les lettres françaises de la fin du XVI ${ }^{e}$ siècle, Genève / Paris, Slatkine, coll. « Bibliothèque Franco Simone », 1992.

BERENGIER Peireto, Fabri de Peiresc. Un segnour e un sage de Prouvènço = Un seigneur et un sage de Provence, Salinelles, L'Aucèu libre, 2015.

BILlIoud Jacques, Le Livre en Provence du XVI $I^{e}$ a XVIII siècle, Marseille, Imp. Saint-Victor, 1962.

BINGEN Nicole et RENAUD Adam (éd.), Lectures italiennes dans les pays wallons à la première Modernité (1500-1630), Turnhout, Brepols, coll. «Études renaissantes », 2015.

BœUF Estelle, Une bibliothèque d'érudit au XVII siècle : les livres de Gabriel Naudé conservés à la Mazarine, Mémoire d'étude, Diplôme de conservateur des bibliothèques, Villeurbanne, ENSSIB, 1998.

BRAGAGLIA Egisto, Gli ex libris italiani dalle origini alla fine dell'Ottocento: Repertorio, vol. II et III, Milan, Editrice bibliografica, 1993.

CAVAlLini Concetta, L'italianisme de Michel de Montaigne, Fasano / Paris, Schena / Presses de l'Université de Paris-Sorbonne, 2003. 
CHARLES-DAUBERT Françoise, «Gabriel Naudé entre la France et l'Italie au temps de Mazarin », dans J. Serroy (éd.), La France et l'Italie au temps de Mazarin, Grenoble, PUG, 1986, p. 100-106.

CHATELAIN Jean-Marc, Un cabinet d'amateur à la fin du XVIII siècle : le marquis de Méjanes bibliophile, Aix-en-Provence, Cité du Livre, 2006.

CHENY Anne-Marie, Une bibliothèque byzantine : Nicolas-Claude Fabri de Peiresc et la fabrique du savoir, Ceyzérieu, Champ Vallon, 2015.

Coninout Isabelle de, «À propos de la bibliothèque aux cotes brunes des Laubespine-Villeroy : les livres italiens chez les secrétaires du roi dans la seconde moitié du $\mathrm{XVI}^{\mathrm{e}}$ siècle ", Italique, vol. VII, 2004, p. 137-159.

D’Amico Silvia, GAMBINo LoNGo Susanna (éd.), Le savoir italien sous les presses lyonnaises à la Renaissance, Genève, Librairie Droz, coll. « Cahiers d'Humanisme et Renaissance », 2017. Dubost Jean-François, La France italienne XVI $-\mathrm{XVII}{ }^{e}$ siècle, Paris, Aubier, coll. « Histoires ", 1997. FERRIGNO Amélie, « Le fonds italien de la Bibliothèque Mazarine : méthodologie, premières données », dans É. Boillet, B. Conconi, C. Lastraioli et M. Scandola (éds), Traduire et collectionner les livres en italien à la Renaissance, Paris, Honoré Champion, 2020, p. 239-250.

FERRIGNO Amélie, «L'italianisme bibliophilique dans le fonds de la Bibliothèque Mazarine ", dans C. Lastraioli (éd.), Poco a poco, l'apport de l'édition italienne dans la culture francophone, Actes du colloque des 27-30 juin 2017, Turnhout, Brepols, à paraître.

FERRIGNO Amélie, «La bibliothèque italienne de Mazarin », dans Y. Loskoutoff, Mazarin, Rome et l'Italie, Actes du colloque des 11-13 mai 2017, Rouen, PURH, à paraître.

Fondation Des banques CIC POUR Le LIVRe et Ministère De LA CUlture, Patrimoine des bibliothèques de France : un guide des régions, vol. 6 : Provence-Alpes-Côte-d'Azur, Corse, Martinique, Paris, Payot, 1995.

FRANKLIN Alfred, Histoire de la bibliothèque Mazarine et du palais de l'Institut, Paris, Welter, 1901. GONTARD Maurice, Histoire de l'Académie d'Aix de 1808 à 1939, Aix-en-Provence, PUP, 1993. JACOB DE SAINT-CHARLES Louis, Traicté des plus belles bibliothèques publiques et particulières, Paris, Rolet Le Duc, 1644.

Jolly Claude, Histoire des bibliothèques françaises, vol. 2 : Les bibliothèques sous l'Ancien Régime, 1530-1789, Paris, Éd. du Cercle de la librairie, 2008.

MiLleR Peter N., L'Europe de Peiresc : savoir et vertu au XVII siècle (préface de M. Fumaroli, traduit de l'anglais par P.-E. Dauzat), Paris, Albin Michel, coll. « L'évolution de l'humanité », 2015.

PALMIRANI Remo, Manuale dell'amatore di ex libris: guida illustrata alla storia, alla cultura ed al collezionismo degli ex libris dal XVI secolo ai giorni nostri, Ravenne, Essegi, 1999.

PÉLIGRY Christian, «La présence italienne dans les collections de la bibliothèque Mazarine principalement au XVII ${ }^{e}$ siècle ", dans D. Montoliu (éd.), Le livre italien hors d'Italie au XVII siècle, Actes du colloque des 23-25 avril 2009, Toulouse, Université Toulouse-Le Mirail, « Collection de l'Écrit », 2011, p. 197-208.

PÉTRARQUE François, Correspondance choisie (lettres choisies et préfacées par P. Laurens, commentaire de U. Dotti mis en français par F. La Brasca), Paris, Les Belles Lettres, 2019.

QUEYROUX Fabienne, Recherches sur Gabriel Naudé, érudit et bibliothécaire du premier XVII siècle (1600-1653), Thèse de l'École nationale des chartes, Paris, 1990. 
Rozzo Ugo et SEIDEL MENCHI Silvana, « Livre et Réforme en Italie », dans J.-F. Gilmont (dir.), La Réforme et le livre. L'Europe de l'imprimé (1517-v. 1570), Paris, Éditions du Cerf, coll. « Histoire », 1990, p. 327-392.

ScHuTZ Alexander H., Vernacular Books in Parisian Private Libraries of the Sixteenth Century according to the Notarial Inventories, Chapel Hill, University of North Carolina Press, 1955.

SERROY Jean (éd.), La France et l'Italie au temps de Mazarin, $15^{\circ}$ Colloque du C.M.R. 17, Grenoble, PUG, 1986.

SORDET Yann, «D’un palais (1643) l'autre (1668) : les bibliothèques Mazarine(s) et leur décor ", Journal des Savants, $\mathrm{n}^{\circ}$ 1, 2015, p. 79-138.

\section{NOTES}

1. Le manuel de comptabilité commerciale est un genre qui s'est particulièrement développé en Italie, notamment grâce au déploiement de l'imprimerie dès la fin $\mathrm{du} \mathrm{xv}^{\mathrm{e}}$ siècle. $\mathrm{Cf}$. A. Ferrigno, "Agostino Chigi et la marchandisation des savoirs. La dédicace de Juan de Ortega ", dans A. Ferrigno, Al Magnifico Agostino Chigi : le mécène et l'imprimerie dans la Rome du XVI e siècle, Presses universitaire de Provence, coll. « Textuelles », 2020.

2. Notamment à travers deux personnages emblématiques de la Provence savante, Pétrarque et Peiresc. Citons entre autres : P. N. Miller (éd.), L'Europe de Peiresc: savoir et vertu au XVII siècle, Paris, Albin Michel, 2015 ; A.-M. Cheny, Une bibliothèque byzantine : Nicolas-Claude Fabri de Peiresc et la fabrique du savoir, Ceyzérieu, Champ Vallon, 2015 ; Pétrarque, Correspondance choisie (lettres choisies et préfacées par P. Laurens, commentaire de U. Dotti mis en français par F. La Brasca), Paris, les Belles lettres, 2019.

3. J. Billioud, Le Livre en Provence du XVI ${ }^{e}$ au XVIII siècle, Marseille, Imp. Saint-Victor, 1962.

4. De nombreuses enquêtes ont été menées au sein des fonds italiens de bibliothèques de France, de Suisse romande et de Belgique, pour retracer les divers circuits du livre italien dans l'espace francophone, approfondir les connaissances sur la réception du livre italien à l'époque moderne en dehors de l'Italie et déterminer sa place au sein des collections privées ou institutionnelles, notamment par le relevé systématique des marques de possession contenues dans les ouvrages. Sur ces enquêtes, cf. <www.editef.univ-tours.fr>.

5. Parmi les études les plus récentes: J. Balsamo, L'amorevolezza verso le cose Italiche. Le livre italien à Paris au XVI e siècle, Genève, Librairie Droz, 2015 ; N. Bingen et R. Adam (éds), Lectures italiennes dans les pays wallons à la première Modernité (1500-1630), Turnhout, Brepols, 2015 ; L. Baldacchini, Il libro e le sue reti, Bologne, Bononia University Press, 2015 ; S. D’Amico et S. Gambino Longo (éds), Le savoir italien sous les presses lyonnaises à la Renaissance, Genève, Librairie Droz, 2017 ; R. Adam et C. Lastraioli (éds), Itinéraires du livre italien à la Renaissance: Suisse romande, anciens Pays-Bas et Liège, Paris, Garnier, 2019 ; C. Lastraioli (éd.), Poco a poco, l'apport de l'édition italienne dans la culture francophone, Actes du colloque des 27-30 juin 2017, Turnhout, Brepols, à paraître.

6. Jean-Baptiste Marie de Piquet (1729-1786), marquis de Méjanes, légua par testament (le 26 mai 1786) sa collection d'environ 80000 livres, constituée à Arles, aux États de Provence «sous la condition d'en tenir une bibliothèque ouverte en la ville d'Aix pour l'avantage du public auquel elle sera destinée ». Cf. Livres en héritage, Bibliothèque Méjanes, dans Fondation des banques CIC pour le livre et Ministère de la Culture, Patrimoine des bibliothèques de France: un guide des régions, vol. 6 : Provence-Alpes-Côte-d'Azur, Corse, Martinique, Paris, Payot, 1995, p. 4.

7. Respectivement : cote C 6878, cote C 5635, cote C 960 .

8. Cote C 767. Cf. Libri italiani, dans Catalogue des livres qui se trouvent chez Fétil, libraire, rue Mazarine, vis-à-vis celle de Guénégaud, au Parnasse Italien, Paris, Fétil, 1781. 
9. Cf. J. Billioud, Le Livre en Provence, ouvr. cité, p. 228-232.

10. Une partie des livres italiens de la bibliothèque Méjanes provient en effet de la bibliothèque du Marquis d'Aubaïs, de la collection de Floncel (ex-libris armorié : « uno dei tre mila libri italiani raccolti da Alberto Francesco Floncel, avvocato nel parlamento di Parigi, consigliere e primo Segretario di Stato del Principato di Monaco nel $1731 »$ ) et de fonds de bibliothèques ecclésiastiques parisiennes.

11. Notons, par exemple, l'ex-libris d'un premier propriétaire italien ou italianisant $d u \mathrm{xvI}^{\mathrm{e}}$ siècle qui fait l'acquisition d'un livre italien à Paris en 1560 : « Costò questo libro 12. co.[...] gl'è a parigi alli 12 di giugno 1560. F. C. P. et amicor [um]» (cote 808450).

12. Cf. A. Ferrigno, "La bibliothèque italienne de Mazarin ", dans Y. Loskoutoff, Mazarin, Rome et l'Italie, Actes du colloque des 11-13 mai 2017, Rouen, PURH, à paraître. Le fonds italien de la bibliothèque Mazarine fut constitué en plusieurs étapes. La première correspond aux achats que Gabriel Naudé (1600-1653) fait pour son maître Jules Mazarin. D'abord, par l'acquisition en bloc de la bibliothèque du chanoine Jean Descordes, puis par de nombreux achats effectués à Paris, auprès des libraires et marchands; et enfin en Italie, lors d'un séjour qui se déroule entre 1645-1646 où Naudé achète plus de 14000 volumes en moins d'un an. La bibliothèque aurait ainsi dépassé les 40000 volumes en 1651 (cf. F. Queyroux, Recherches sur Gabriel Naudé, érudit et bibliothécaire du premier XVII siècle (1600-1653), Thèse de l'École nationale des chartes, Paris, 1990). Par la suite, le fonds italien est également enrichi par les échanges de 1668 avec la bibliothèque royale (une partie de ces livres provient également de la bibliothèque de Gaston d'Orléans et peut contenir des marques de provenance antérieures) ainsi que par divers ouvrages issus de récupérations révolutionnaires de bibliothèques d'établissements ecclésiastiques parisiens confisquées, ayant elles-mêmes été enrichies par l'apport de collections domestiques privées, de legs, de dons, ou d'achats.

13. $\mathrm{Cf}$. A. Ferrigno, «Le fonds italien de la Bibliothèque Mazarine: méthodologie, premières données ", dans É. Boillet, B. Conconi, C. Lastraioli et M. Scandola (éds), Traduire et collectionner les livres en italien à la Renaissance, Paris, Honoré Champion, 2020, p. 239-250; A. Ferrigno, «L'italianisme bibliophilique dans le fonds de la Bibliothèque Mazarine », dans C. Lastraioli (éd.), Poco a poco, l'apport de l'édition italienne dans la culture francophone, ouvr. cité.

14. Cote C 2809. La localisation de « Monlunello» reste inconnue.

15. Cote Res D 91518.

16. Voir les travaux de J.-M. Chatelain, «Le goût des livres rares : la collection du marquis de Méjanes en son temps » (communication tenue dans le cadre du bicentenaire de la bibliothèque Méjanes), Aix-en-Provence, 18 novembre 2010 et Id., Un cabinet d'amateur à la fin du XVIII siècle : le marquis de Méjanes bibliophile, Aix-en-Provence, Cité du Livre, 2006.

17. M. Gontard, Histoire de l'Académie d'Aix de 1808 à 1939, Publications de l'Université de Provence, 1993.

18. On compte environ 26000 imprimés cotés au sein de la bibliothèque Paul Arbaud, et autant d'ouvrages non cotés : <https://museebibliographique-arbaud.centredoc.fr>.

19. Cf. M. Gontard, Histoire de l'Académie d'Aix, ouvr. cité, p. 181-185.

20. Ces ouvrages figurent tous dans l'inventaire de la bibliothèque.

21. La rareté des exemplaires explique sans doute ce manque, mais la bibliothèque Arbaud et ses inventaires n'ont pas encore révélé tous leurs trésors.

22. Cote R 0517 : Jean Pellicot, Traicté de l'Église de Dieu contre les Calvinistes, Aix, Pierre Roux, 1575.

23. Cote S 385 : Michel Nostradamus, Almanach pour l'an M.D.LXIII avec les présages, calculé [et] expliqué par M. Michel Nostradamus, docteur en médicine, aftrophile de Salon de Craux en Provence. (Dédié) [...] "Allo illustrissimo Signore, il S. Fran Fabritio de Serbolloni, per sua Sātità nelle cose della guerra in Francia Generale" par M. Nostradamus. Fabrice Serbelloni », s. 1., 1563.

Cote R 736: Jean de Notre Dame, Le vite delli più celebri et antichi primi poeti provenzali, Lyon, Alessandro Marsili, 1575.

24. Cote R 2002 : Le metamorfosi di Ovidio [...] con annotationi di M. Gioseppe Horologgi, Venise, 1584. 
25. Cote R 240: Dante con nuove et utili ispositioni aggiuntovi di più una tavola di tutti $i$ vocaboli più degni d'osservatione [...], Lyon, Guglielmo Rouillio, 1551.

26. Cote Q 275 : Arioste, Orlando furioso, Venise, 1603.

27. Cote R 113 : Lettere della Signora Isabella Andreini Padovana comica gelosa, Venise, Gio. Battista Combi, 1620.

28. Cote R 272 : Battista Guarini, Le Berger fidelle traduit de l'italien de Guarini en vers françois [par l'abbé de Torche], avec le texte italien en regard, Cologne, Philippe du Marteau, 1671.

29. Cote R 521 : Vasquin Philieul, Laure d'Avignon au nom et adveu de la Royne Catharine de Medicis Royne de France, Extraict du poete florentin Françoys Petrarque et mis en Françoys par Vasquin Philieul de Carpentras, Paris, Jacques Gazeau, 1548. L'exemplaire porte l'ex-libris de Paul Arbaud : « Ex-libris Paul Arbaud mi fan gau ».

Cote R 2003: Giacomo Filippo Tomasini, Petrarcha redivivus: integram poetae celeberrimi vitam iconibus aere caelatis exhibens [...], Patavii, Pauli Frambotti bibliopolae, 1650.

30. Cote R 645 : Fantoni Castrucci, Istoria della città d'Avignone e del contado venesino, s. l., 1678. L'exemplaire porte l'ex-libris de Paul Arbaud : «Ex-libris Paul Arbaud mi fan gau ».

31. Cote R 509 : Giorgio de Rusconi, La conversione di Sancta Maria Madalena, s. 1., 1517, ainsi que les cotes S 430, S 431, S 432, S 433, S 445, S 465, S 484, S 485. Les ouvrages de théâtre et de poésie magdalénienne portent les cotes S 452, S 457, S 458, S 459, S 466, S 471, S 474.

32. O. de Saint-Gelais, La Chasse d'amours: poème publié en 1509, éd. critique par M. B. Winn, Genève, Librairie Droz, 1984, p. Ix.

33. Dans son allocution d'ouverture de la première séance de l'Académie à l'hôtel Arbaud, le président Bonnecorse désigne la collection de Paul Arbaud (bibliothèque et musée) comme « un monument magnifique consacré à la gloire de [la] Provence " (M. Gontard, Histoire de l'Académie d'Aix, ouvr. cité, p. 185).

34. Cote D 3209.

35. Cote Q 893.

36. Cote R 448 .

37. Cf. I. de Conihout, «À propos de la bibliothèque aux cotes brunes des Laubespine-Villeroy : les livres italiens chez les secrétaires du roi dans la seconde moitié du XVI ${ }^{\mathrm{e}}$ siècle », Italique, vol. VII, 2004, p. 137-159. Quelques exemples : $15 \%$ de livres en langue italienne dans la bibliothèque de Nicolas Peiresc (1580-1637) sur 6000 volumes; 9,3\% de la bibliothèque de Jean Descordes (1570-1642) sur 6000 volumes environ; 10,6\% de la bibliothèque du cardinal de Richelieu (1585-1642) sur 6000 volumes environ; $6 \%$ dans la bibliothèque de Claude Dupuy (1545-1594); $6 \%$ de la bibliothèque de Jacques-Auguste de Thou (1553-1617) sur 6000 volumes ; $10 \%$ des titres reliés pour Grolier ; $25 \%$ des 120 titres recensés ayant appartenu à Thomas Mahieu (1515-1588).

38. La notion d' «italianisme bibliophilique " est à manier avec prudence pour l'étude des possesseurs de cette époque. Cette mode pratiquée depuis les guerres d'Italie, renforcée et encouragée par une forte présence italienne à Paris - de libraires et marchands, mais surtout d'«Italiens français » séjournant à la cour, ou faisant partie des milieux d'affaires - depuis François $\mathrm{I}^{\mathrm{er}}$, l'italianisme n'a eu de cesse de se développer dans la production artistique, littéraire, scientifique et éditoriale française, contribuant à la circulation du livre italien à Paris. Sur cette notion, cf. A. Ferrigno, «L'italianisme bibliophilique dans le fonds de la Bibliothèque Mazarine », dans C. Lastraioli (éd.), Poco a poco, l'apport de l'édition italienne dans la culture francophone, ouvr. cité. Voir également l'exposition virtuelle «Libri italiani / Lecteurs français " sur le site de la bibliothèque Mazarine: <www.bibliotheque-mazarine.fr/fr/bibliotheque-numerique/ expositions-virtuelles/libri-italiani-lecteurs-francais-exposition-virtuelle>. 


\section{RÉSUMÉS}

Dans le cadre du projet EDITEF (L'édition italienne dans l'espace francophone à la première modernité), plusieurs fonds italiens de bibliothèques françaises ont été examinés. L'enquête permet de collecter un grand nombre de données qui nous renseignent sur les différentes voies de circulation des livres en langue italienne en France, notamment au moyen de l'examen des marques de provenance laissées par les divers possesseurs. Quelle vision de l'Italie recherchaientils, choisissaient-ils, à travers quels livres (auteurs, genres...) et pour quels besoins (apprentissage, échanges scientifiques, intellectuels, diplomatiques...)?

Les marques de provenance contenues dans les fonds italiens de la bibliothèque Méjanes et de la collection Paul Arbaud nous éclairent sur la réception du livre en langue italienne en Provence, et sur la façon dont la "culture italienne " pénètre l'espace francophone depuis le $\mathrm{XVI}^{\mathrm{e}}$ siècle, dans un contexte où les échanges politiques et militaires se mêlent au développement d'un italianisme artistique, scientifique, littéraire, éditorial et « bibliophilique », pour parvenir jusque dans les bibliothèques de collectionneurs plus récents.

Il progetto EDITEF (L'edizione italiana nello spazio francofono nella prima età moderna) ha permesso di studiare più fondi italiani conservati in biblioteche francesi. L'indagine consente di raccogliere un gran numero di dati, che ci informano sulle diverse modalità di circolazione dei libri in lingua italiana in Francia, specialmente attraverso l'esame degli ex libris lasciati dai diversi possessori. Quale immagine dell'Italia ricercavano, sceglievano, attraverso quali libri (autori, generi...) e per quali esigenze (apprendimento linguistico, scambi scientifici, intellettuali, diplomatici...)?

Gli ex libris contenuti nei fondi italiani della Bibliothèque Méjanes e della collezione Paul Arbaud evidenziano le vie della ricezione del libro in lingua italiana in Provenza, e il modo in cui la «cultura italiana» è penetrata nello spazio francofono a partire dal sedicesimo secolo, in un contesto in cui gli scambi politici e militari si sono mescolati con lo sviluppo di un «italianismo» artistico, scientifico, letterario, editoriale e «bibliofilo», per giungere poi nelle biblioteche di collezionisti più recenti.

For the EDITEF project (Italian Books and Book Collections in Early Modern French-Speaking Countries), several Italian collections from French libraries were examined. The survey made it possible to collect a large amount of data informing on the different circulation routes of the Italian books in France, in particular by examining the marks of provenance left by the various owners. What vision of Italy were they seeking, choosing, through which books (authors, genres...) and for which purposes (scholarly, intellectual, or diplomatic exchanges, learning...)?

The ex libris contained in the Italian collections of the Bibliothèque Méjanes and the Paul Arbaud collection shed light on the reception of the Italian book in Provence, and on how "Italian culture" has penetrated the French-speaking world since the 16th century, in a context where political and military exchanges mixed with the development of artistic, scientific, literary, editorial and bibliophilic Italianism to reach the libraries of more recent collectors. 
INDEX

Keywords : library, Mazarine, Méjanes, Paul Arbaud, Italian books, Renaissance, owner, ex libris, dissemination, Paris, Aix-en-Provence, EDITEF, edition

Parole chiave : biblioteca, Mazarine, Méjanes, Paul Arbaud, libri italiani, Rinascimento, possessori, ex libris, circolazione, ricezione, Parigi, Aix-en-Provence, EDITEF, editoria Mots-clés : bibliothèque, Mazarine, Méjanes, Paul Arbaud, livres italiens, Renaissance, possesseurs, ex-libris, circulation, diffusion, Paris, Aix-en-Provence, EDITEF, édition

\section{AUTEUR}

AMÉLIE FERRIGNO

CESR, EDITEF, Bibliothèque Paul Arbaud 